\title{
Web-based Platform for Training in Biomedical Signal Processing and Classification: the Particular Case of EEG-based Drowsiness Detection
}

\author{
https://doi.org/10.3991/ijoe.v14i03.8193 \\ Daniel Ribeiro, César Teixeira, Alberto Cardoso $\left.{ }^{(}\right)$ \\ University of Coimbra, Coimbra, Portugal \\ alberto@dei.uc.pt
}

\begin{abstract}
Online Experimentation, which comprises remote and virtual experimentation, has played an important role, over the years, in the development of the students learning process. One of its examples is the use of webbased resources to study physiological events in topics of biomedical engineering. This article discusses the use of a virtual experimentation environment as a training tool for studying different methodologies that can be applied to detect drowsiness based on electroencephalogram (EEG) signals. As a result of this web-based platform, students can be more motivated to learn different methods that can be used in the processing and analysis of physiological data. As a final result, it can even be used to create a real-time detector for drowsiness, thus preventing the occurrence of road accidents.
\end{abstract}

Keywords-Drowsiness, Electroencephalogram (EEG), Web-based resources, Online experimentation

\section{Introduction}

According to a study carried by Driver et al. in 1994, the difficulties in understanding scientific concepts are present in all ages and levels [1]. A research done by Rocard [2] concluded that new tools and methodologies are needed, so that the learning process in schools can become more efficient and motivating. Because we live in a time with a rapid development of technology, the "traditional" education has surpassed to something new. Nowadays, classroom activities and demonstrations can be done by the use of online laboratories $[3,4]$, considering essentially real or virtual experiments and digital tools, which provide students with new opportunities to explore and understand different scientific concepts. In fact, a study carried by Dikke et al. [5] shows that many European schools are equipped with computer classes, tablets and high-speed internet connection for use of a huge variety of web-based learning applications and simulations. Another study, conducted by Zacharia [6], indicates that the simultaneous use of real and virtual experimentation improves students' conceptual understanding, comparing with the use of real experimentation alone. 
This article presents a web-based platform that aims to improve the learning process of biomedical engineering students on signal processing and classification. Here, the drowsiness detection based on EEG signals is taken as an example.

Sleep can be categorized into two types, rapid eye movement (REM) and non-rapid eye movement (NREM). The latter can be further divided into 4 stages: 1, 2, 3 and 4 [7]. In this work, the focus is into stage 1 because it corresponds to the transition period between awareness and sleepiness, the case study considered in this paper. At this stage, the brain waves and activities begin to slow down, translating into decreased EEG signal frequency and, in some cases, contractions called hypnagogic myoclonus may occur, preceded by a falling sensation. This stage has an average duration of 1 to 7 minutes in the initial cycle [7].

The paper is organized as follows: Section 2 describes the methods developed in this work, regarding the signal processing and classification, as well as how to use the web platform. Section 3 is devoted to presenting the results obtained with the different methodologies. Finally, in Section 4 some conclusions are drawn.

\section{Methods}

\subsection{Signal processing $\&$ classification pipeline}

The developed platform uses Matlab as processing engine. The implemented processing steps are shown in Figure 1. First, these signals were filtered with a band-pass Butterworth filter, with a pass-band from 0.3 to $30 \mathrm{~Hz}$. This frequency range was chosen since outside it we have no biological meaning for the physiological process in question: below $0.3 \mathrm{~Hz}$, we have the presence of artifacts from other signals and in the $50 \mathrm{~Hz}$ range, the presence of noise.

In a next step, the features were extracted, using different window sizes and increments, for further study. Having extracted the amount of 13 features, the most representative ones were selected, using appropriate selection methods. In total, five selection methods were used. It has been decided that a feature is considered relevant to the problem if it is selected as relevant by at least two methods. The features were extracted from the time and frequency domains. From the time domain, mean, variance, asymmetry, kurtosis and Hjorth coefficients (complexity and mobility) were extracted. As features from the frequency domain, entropy, average power, power spectrum density and relative power in conventional EEG sub-bands were taken into account. Later, the use of Principal component Analysis as a feature reduction method was studied.

Afterwards, the training set was equalized and normalized. Equalization means that, as there are much less patterns from the drowsiness class than from the nondrowsiness class, it is necessary to eliminate non-drowsiness patterns in order to have at the end the same number of patterns for both classes in the training set. It should be noted that the test was performed with original data, without any pattern elimination, to properly evaluate the detection performance. In addition, testing was conducted in a causal process, which means that only actual and past information was used. 
In the final step, the introduction of delay in the input data was studied, and then different classifiers were created and trained so that they could be used later in the web platform. Regarding classification, classifiers were trained to identify the drowsiness and non-drowsiness states.

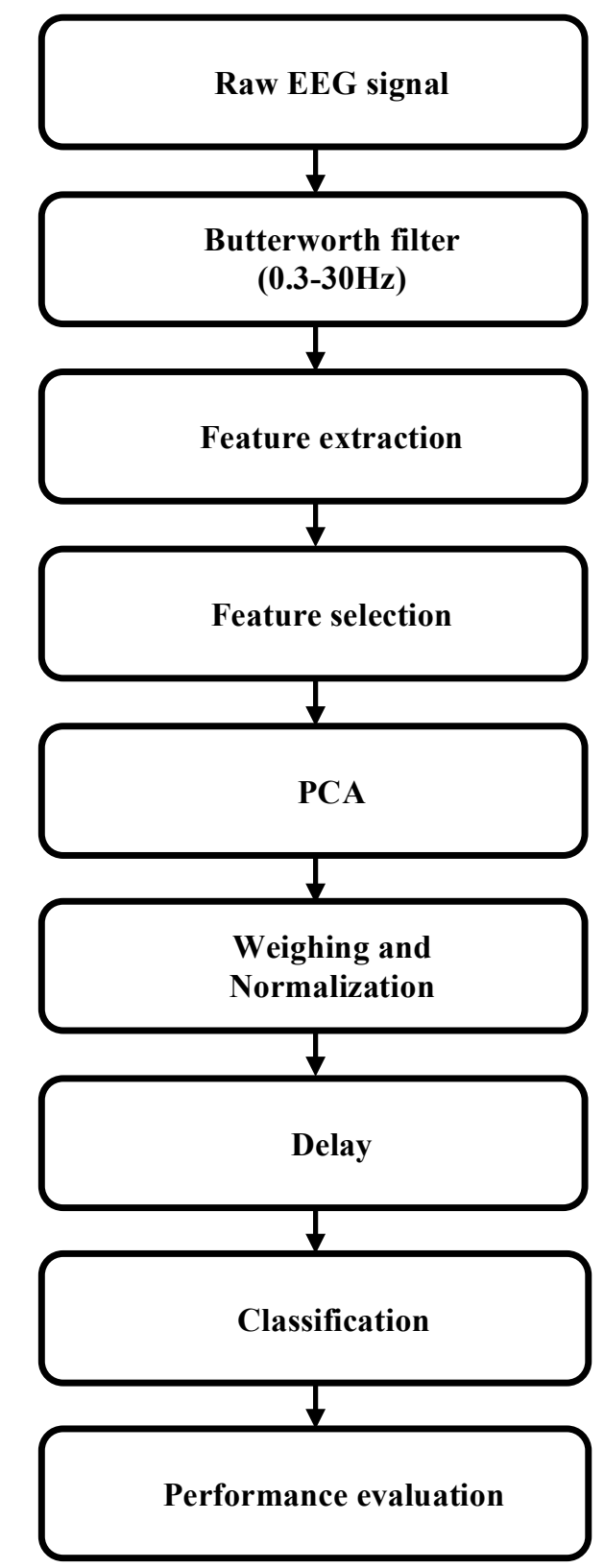

Fig. 1. Processing steps implemented for drowsiness detection. 


\subsection{Web Platform}

Development. Has already mentioned, the web platform, shown in Figure 2, was developed with the purpose of helping students to study and understand the best methodologies to detect situations of drowsiness, thus offering an initial help in the development of their work through a different learning process. Additionally, with this platform, students can improve their sensitivity on the impact of certain methodologies used in signal analysis and processing.

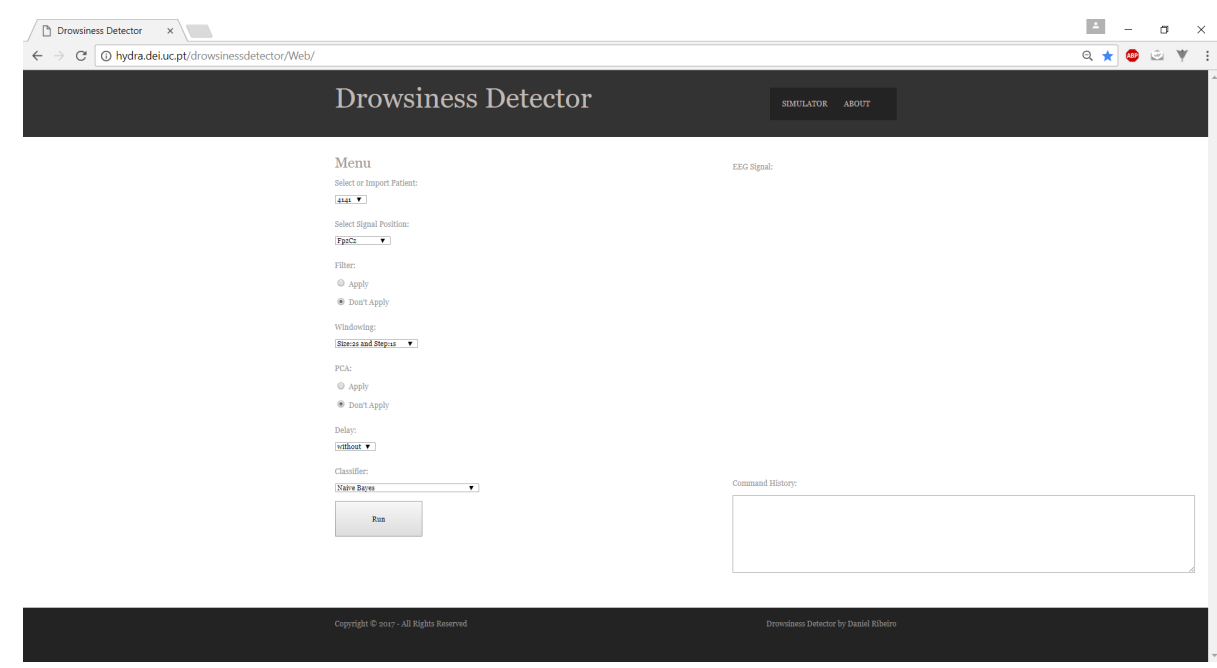

Fig. 2. Web platform developed to study methods for the detection of drowsiness.

Regarding the web platform, on the server side, Java was used, while on the client side, several web languages were considered, such as Hypertext Markup Language (HTML), JavaScript, among others. More precisely, all the structuring and presentation of the content is done through HTML, along with the Cascading Style Sheets (CSS), where the styles (colors, fonts, spacing, etc.) of the web document are defined. The purpose of CSS is to store all information related to the project, rather than being placed in HTML, making the project more structured and organized.

Also in the client-side, JavaScript was used, which is "triggered" when the user presses the Run button, using Asynchronous JavaScript and XML (AJAX) to make asynchronous requests. With AJAX, the web platform can send requests to the server and receive them asynchronously (in the background) without interfering with the display and behavior of the existing webpage. By separating the submission layer from the presentation layer, AJAX allows webpages to dynamically change their content without the need to reload the entire page. To facilitate the use of AJAX, the JavaScript Object Notation (JSON) is adopted. To simplify the code in the created script, the jQuery, a JavaScript function library, was used.

Through the AJAX request, where data related to the selected classifier, such as the position of the EEG signal, among other options, can be defined, the connection is established with the server that, as previously referred, uses Java. Here, the MatCon- 
soleCtl library, available in Github, is used, which basically allows making calls to Matlab from Java. This library establishes a session with Matlab, allowing the call of certain functions. These functions were developed in simulation mode, with the return values being sent to the client-side. They are then presented as a graph with the EEG signal(s) and the results of the classification, showing the performance of the selected methodology. This mechanism is illustrated, in synthesis, in Figure 3.

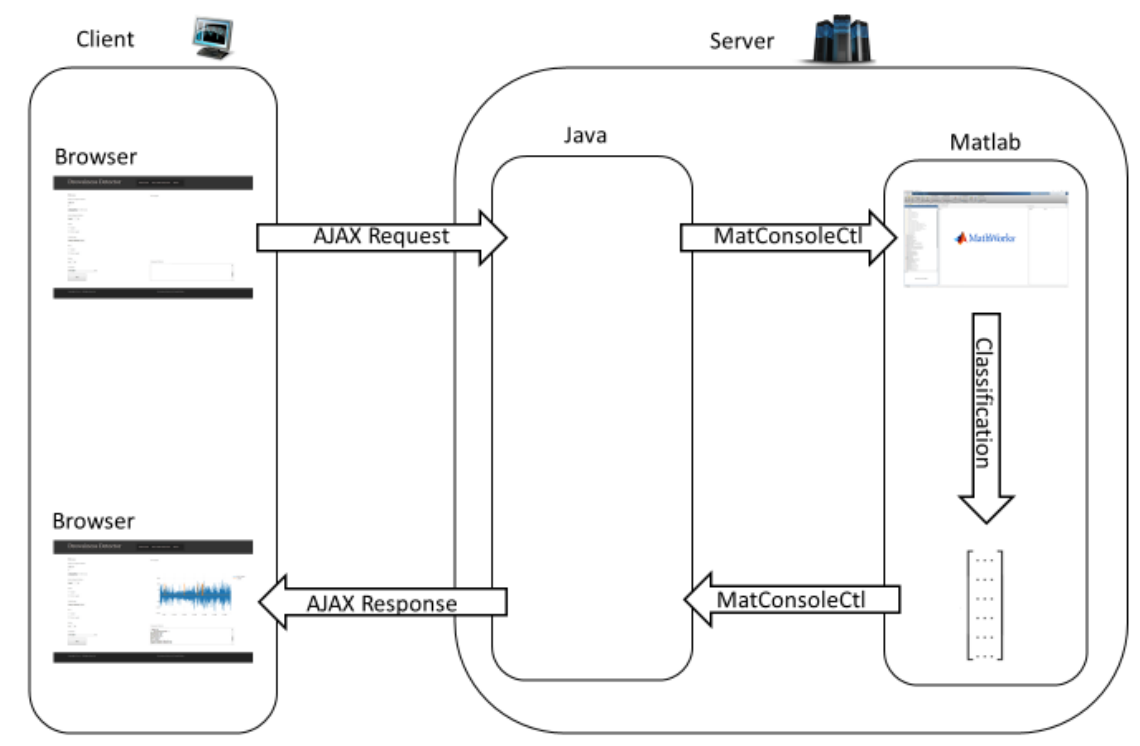

Fig. 3. Mechanism describing the operation of the web platform.

\subsection{Use of the web platform}

In the web platform, the user has the possibility to choose an EEG signal from the available signal set, which can vary in the position(s) and be of different persons. To filter the signal, according to Figure 4, the following parameters/options have to be chosen: the width and the step of the sliding windows; the application of PCA; the application of delay selecting one of 5 different values; and select the classifier.

After choosing these options and pressing the Run button, the mechanism, previously described, is activated for about 40 seconds. Simultaneously, information regarding the user's choices appears in the Command History, as shown in Figure 5. At the end, a graph with the EEG signal(s) and their classifications is presented, as well as the features used for the classification process and the performance results of the selected methodology (specificity, sensitivity, and others), in the Command History. Regarding the graph, the user can manipulate it, that is, it can be zoomed in/out to analyze a specific part of the signal and, also, save the picture. 


\section{Drowsiness Detector}

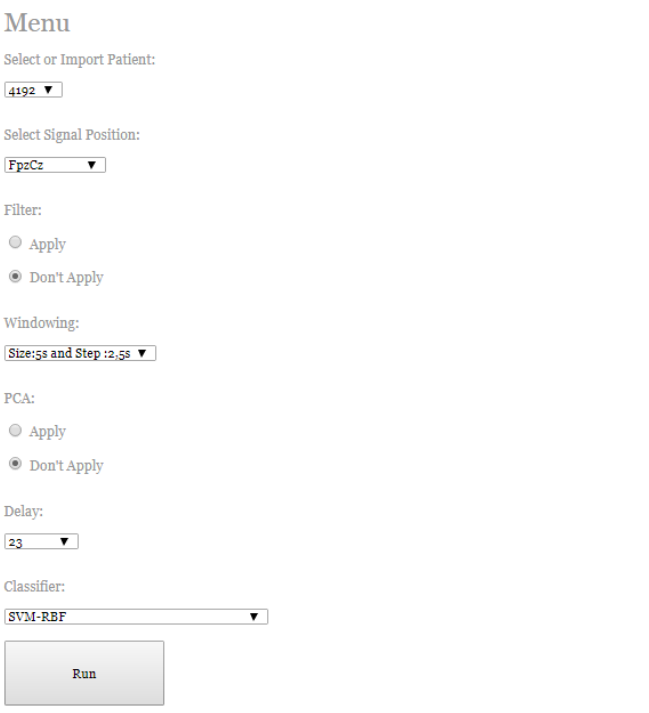

\section{Copyright $\odot 2017$ - All Rights Reserved}

Fig. 4. Available options for user selection.
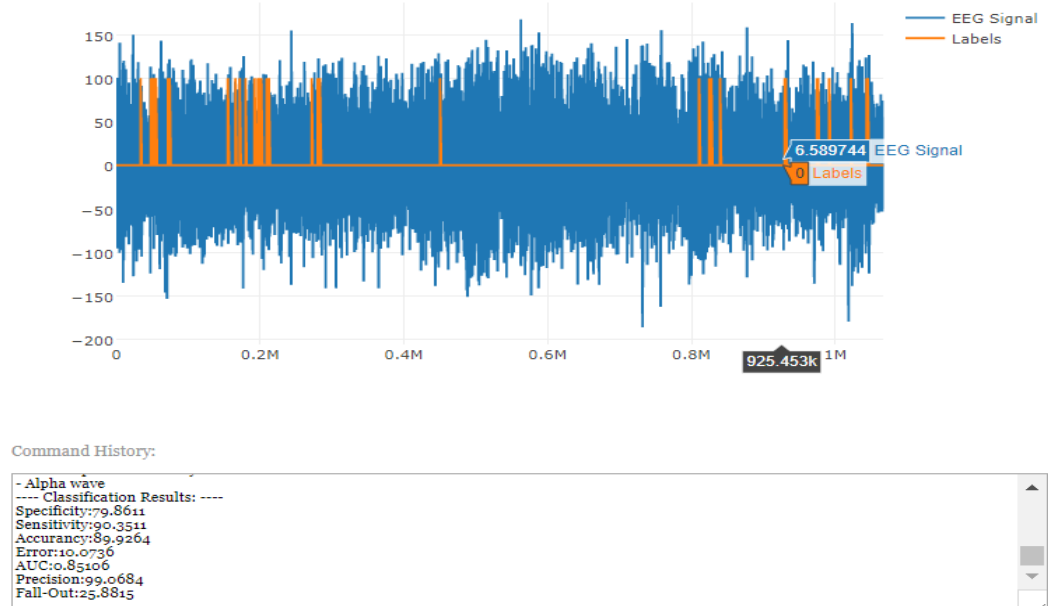

Fig. 5. Example of using the web platform for drowsiness detection. 


\section{Results}

In this article, the database considered to test and evaluate the application of the methodologies and the use of the web platform was retrieved from Physionet [8], and is titled "The Sleep-EDF Database [Expanded]". This database consists of 39 polysomnograms, with an average duration of 20 hours, with the respective hypnograms and annotations of the different sleep stages of healthy individuals. As the intention was to use only EEG signals, the other signals present in the polysomnograms were omitted.

Regarding the different methodologies considered throughout this study, Table 1 shows the results obtained with the best ones. As can be observed, the best results were obtained with the use of delays, specifically with the 23 and 12 combinations, in which the second and third previous epochs, and the past and second previous epochs, respectively, were used. In all cases, classifiers based on Support Vector Machines (SVM) with radial basis function (RBF) kernel were adopted. With these approaches, classifications with about $99.5 \%$ of precision and $90 \%$ of accuracy were obtained.

Table 1. Best results obtained for the detection of drowsiness.

\begin{tabular}{|c|c|c|c|c|c|c|}
\hline Classifier & Window Information & Signal Position & PCA & Delay & Precision (\%) & Accuracy (\%) \\
\hline SVM (RBF) & $\begin{array}{c}\text { Size: } 5 \mathrm{~s} \\
\text { Step: } 2.5 \mathrm{~s}\end{array}$ & Fpz-Cz & $\mathrm{N}$ & 23 & 99.58 & 89.60 \\
\hline SVM (RBF) & $\begin{array}{c}\text { Size: } 5 \mathrm{~s} \\
\text { Step: } 2.5 \mathrm{~s}\end{array}$ & Fpz-Cz & $\mathrm{N}$ & 12 & 99.58 & 89.45 \\
\hline SVM (RBF) & $\begin{array}{c}\text { Size: } 10 \mathrm{~s} \\
\text { Step: } 2 \mathrm{~s}\end{array}$ & Fpz-Cz & $\mathrm{N}$ & 23 & 99.55 & 89.27 \\
\hline SVM (RBF) & $\begin{array}{c}\text { Size: } 5 \mathrm{~s} \\
\text { Step: } 2.5 \mathrm{~s}\end{array}$ & Fpz-Cz & $\mathrm{N}$ & 12 & 99.54 & 90.02 \\
\hline
\end{tabular}

\section{Conclusions}

This article presents a web-based platform that is the front-end of a system that allows the processing and classification of biomedical signals. In this work, the problem of drowsiness detection based on EEG signals was studied, considering the database "The Sleep-EDF Database [Expanded]" from Physionet to compare and evaluate the application of different methodologies for classification purposes. According to the results, classifiers based on SVM with RBF kernel presented the best performance for the detection of drowsiness.

The described web platform provides an alternative environment for the students' learning process, contributing to better understanding the role of different classification methods for the processing and analysis of physiological data.

It is expected that this approach can be used to create a real-time drowsiness detector, contributing, for example, to prevent the occurrence of road accidents due to the drowsiness of drivers. 


\section{$5 \quad$ References}

[1] Driver, R., Leach, J., Scott, P., Wood-Robinson, V. (1994). Young people's understandding of science concepts: implications of cross-age studies for curriculum planning. Studies in Science Education, 24, 75-100. https://doi.org/10.1080/03057269408560040

[2] Rocard, M. (2007). Science education NOW: a renewed pedagogy for the future of Europe. Luxembourg: Office for Official Publications for the European Commission. Available at: http://ec.europa.eu/research/science-society/document library/pdf 06/reportrocard-on-science-education en.pdf.

[3] Cardoso, A., Vieira, M., Gil, P. (2012), A Remote and Virtual Lab with Experiments for Secondary Education, Engineering and Lifelong Learning Courses. International Journal of Online Engineering (iJOE), 8(Special Issue: exp.at'11): 49-54. https://doi.org/10.3991/ ijoe.v8iS2.1984

[4] Sánchez, J., Morilla, F., Dormido, S., Aranda, J., Ruipérez, P. (2002). Virtual and Remote Control Labs Using Java: A Qualitative Approach. IEEE Cont. Syst. Mag. 22 (2): 8-20. https://doi.org/10.1109/37.993309

[5] Dikke, D., Tsourlidaki, E., Zervas, P., Cao, Y., Faltin, N., Sotiriou, S., Sampson, D. (2014). GOLABZ: Towards a federation of online labs for inquiry based science education at School. Proceedings of the 6th International Conference on Education and New Learning Technologies (EDULEARN2014).

[6] Zacharia, Z.C. (2007). Comparing and combining real and virtual experimentation: an effort to enhance students' conceptual understanding of electric circuits, Journal of Computer Assisted Learning, 23: 120-132. https://doi.org/10.1111/j.1365-2729.2006.00215.x

[7] Colten, HR., Altevogt, BM. (2006). Sleep Disorders and Sleep Deprivation: An Unmet Public Health Problem. Institute of Medicine, National Academies Press (US).

[8] Goldberger AL, Amaral LAN, Glass L, Hausdorff JM, Ivanov PCh, Mark RG, Mietus JE, Moody GB, Peng C-K, Stanley HE. (2000). PhysioBank, PhysioToolkit, and PhysioNet: Components of a New Research Resource for Complex Physiologic Signals. Circulation. 101(23): e215-e220. https://doi.org/10.1161/01.CIR.101.23.e215

\section{Authors}

Daniel Ribeiro received the M.Sc. degree in Biomedical Engineering from the University of Coimbra, Portugal. (email: danieltaribeiro@gmail.com)

César Teixeira received the Ph.D. degree in Electronics Engineering and Informatics from the University of the Algarve, and is with the Department of Informatics Engineering and the Centre for Informatics and Systems of the University of Coimbra (CISUC). (email: cteixei@dei.uc.pt)

Alberto Cardoso received the Ph.D. degree in Informatics Engineering from the University of Coimbra, and is with the Department of Informatics Engineering and the Centre for Informatics and Systems of the University of Coimbra (CISUC). (email: alberto@dei.uc.pt)

Article submitted 03 January 2018. Resubmitted 26 February 2018. Final acceptance 05 March 2018. Final version published as submitted by the authors. 